Shack-Hartmann Wavefront Sensing Performance Evaluation for Active Correction of the Large Synoptic Survey Telescope (LSST)

K. L. Baker, L. Seppala

July 30,2010

SPIE Optics and Photonics

San Diego, CA, United States

August 1, 2010 through August 5, 2010 
This document was prepared as an account of work sponsored by an agency of the United States government. Neither the United States government nor Lawrence Livermore National Security, LLC, nor any of their employees makes any warranty, expressed or implied, or assumes any legal liability or responsibility for the accuracy, completeness, or usefulness of any information, apparatus, product, or process disclosed, or represents that its use would not infringe privately owned rights. Reference herein to any specific commercial product, process, or service by trade name, trademark, manufacturer, or otherwise does not necessarily constitute or imply its endorsement, recommendation, or favoring by the United States government or Lawrence Livermore National Security, LLC. The views and opinions of authors expressed herein do not necessarily state or reflect those of the United States government or Lawrence Livermore National Security, LLC, and shall not be used for advertising or product endorsement purposes. 


\title{
Shack-Hartmann Wavefront Sensing Performance Evaluation for Active Correction of the Large Synoptic Survey Telescope (LSST)
}

\author{
K.L. Baker*, L. Seppala \\ Lawrence Livermore National Laboratory, 7000 East Ave., Livermore, CA, USA 94550
}

\begin{abstract}
The Large Synoptic Survey Telescope will map out the dark matter in the universe and is scheduled to see "first light" in 2014. This telescope will require active correction of its mirrors to remove the aberrations that arise from changing gravitational force vectors and from thermal drifts in the telescope during observational runs. In this article we present a comprehensive evaluation of a Shack-Hartmann wavefront sensor and reconstruction algorithm which is capable of meeting the unique challenges associated with this wide field-of-view survey telescope. The advantages of this technique over other potential wavefront sensing technologies are discussed and the potential problems encountered with this approach are analyzed and solutions to these problems presented.
\end{abstract}

Keywords: Adaptive optics, Hartmann, wave-front sensing

\section{INTRODUCTION}

The Large Synoptic Survey Telescope (LSST) is a three mirror, ground-based, wide field-of-view, survey telescope in a Paul-Baker configuration.[SPIE paper] One of the features that distinguishes this telescope is its large etendue, 318 $\mathrm{m}^{2} \mathrm{deg}^{2}$, which is nearly two orders of magnitude higher than existing telescopes. The telescope will view a 3.5 degree patch of sky with a 3.5 gigapixel focal plane array every 15 seconds. This cadence will enable the LSST to map out the entire sky every three nights. This unprecedented capability will open up many new exciting scientific capabilities.

The scientific objectives of the LSST include mapping the distribution of dark matter and dark energy using weak gravitational lensing, strong gravitational lensing, supernova cosmology, quasar detection and an all-sky photometric redshift survey.[LSST Proposal] Measuring weak lensing as a function of redshift provides information on the evolution of structure in the universe as a function of cosmic time. Precision measurements of the shear power spectra vs cosmic time will constrain the sum neutrino mass. The baseline operation of the LSST survey is expected to discover $\sim 250,000$ Type 1a supernova per year. LSST will enable the detection of quasars through their temporal variability and it is expected to provide a tenfold increase in the number of known quasars. The all-sky photometric redshift survey will enable cross-correlation between LSST data sets and with detailed maps of the structure of the cosmic microwave background. By simultaneously measuring mass growth and curvature, LSST can elucidate whether the recent acceleration is due to dark energy or modified gravity.

The success of the scientific mission involving the measurement of weak lensing depends upon the ability to measure extremely small ellipticity correlations in the observed galaxies. This in turn places requirements on the telescope to ensure that the mirrors of the telescope do not affect the ellipticity correlation measurements of the multitude of galaxies captured on the focal plane array. To measure the wavefront error due to both residual atmospheric turbulence and changing telescope mirror deformations, wavefront sensors will be placed on the focal plane array to measure the aberrations and based upon those measurements to correct for the mirror deformations. The measurements will be integrated in time for 15 seconds such that all but the lower frequency atmospheric aberrations will be averaged out over the course of the measurement.

In this article we review the design and function of the Large Synoptic Survey Telescope, along with its novel wavefront sensor geometry and unique requirements in the following section. In the third section we review the ShackHartmann wavefront sensors in general and in particular the design used to evaluate their use on the LSST. In the fourth section we review the expected fluence levels which will be available for wave-front sensing in the various bands and sky locations. In the fifth section we discuss the reconstruction algorithm that we use to reconstruct the phase across the LSST pupil. We evaluate the expected performance of the wave-front sensors under the postulated conditions of operation in section six and discuss their full implications in section 7 . We then summarize the result in the eighth and final section. 


\section{LARGE SYNOPTIC SURVEY TELESCOPE (LSST)}

The Large Synoptic Survey Telescope (LSST) consists of three mirrors in a Paul-Baker design to enable a wide field-ofview, 3.5 degrees, on the sky. The telescope is scheduled to be built at Cerro Pachon and have first light in 2014. The telescope is shown below in Figure 1. In addition to the three mirrors, there are also three lenses and an array of filters which are used to correct and flatten the focal plane and to select the desired wavelength band, respectively. Each of the three mirrors on the telescope will be actively controlled; a monolithic tertiary $(5.2 \mathrm{~m}) /$ primary $(8.4 \mathrm{~m})$ mirror made of honeycomb borosilicate and a $3.4 \mathrm{~m}$ diameter secondary mirror. To carry out its science missions, the LSST will also operate with six different wavelength filter bands; U(330-400 nm) G(402-552 nm), R(552-691 nm), I(691-818 nm), $\mathrm{Z}(818-922 \mathrm{~nm})$ and $\mathrm{Y}(950-1060 \mathrm{~nm})$.

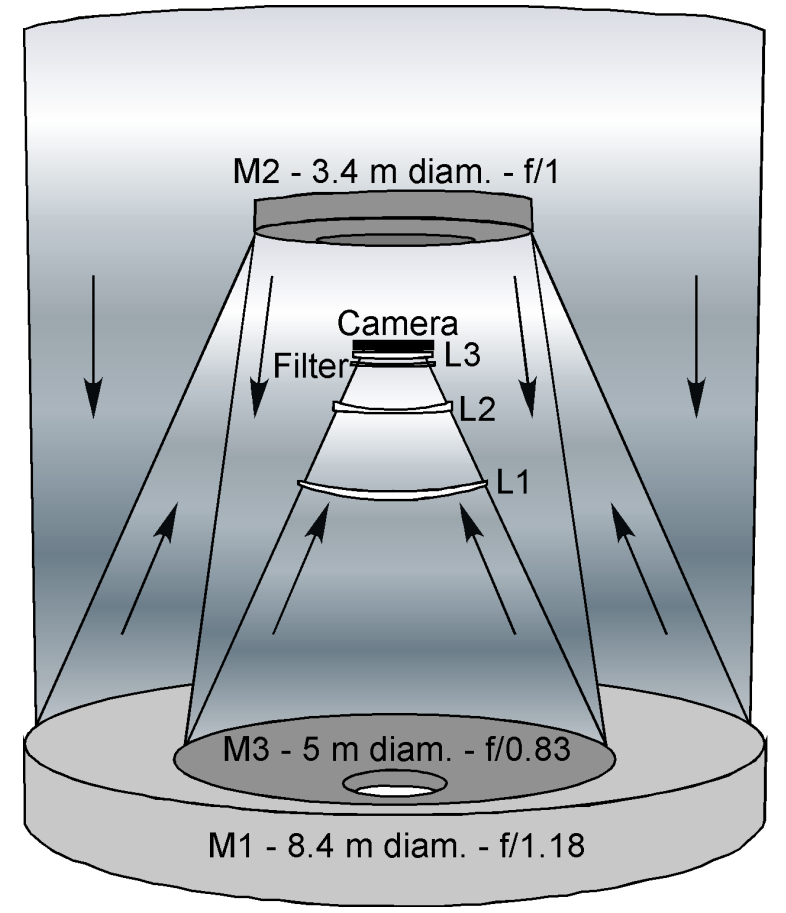

Figure 1 Layout of the Large Synoptic Survey Telescope (LSST). The LSST contains three mirrors in a Paul-Baker configuration to enable a reasonably well corrected 3.5 degree field-of-view across the focal plane array.

Because there are three separate mirrors which must be controlled, this telescope requires multiple wave-front sensors in the focal plane to tomographically reconstruct the phase at each of the mirrors. As the telescope surveys the night sky taking 15 second exposures, the mirrors will experience differing force vectors due to gravity and will have to be adjusted for changes in temperature over the course of the night. A separate article has been written to address the issue of tomographic reconstruction of the mirror surfaces given a perfect wave-front sensor.[Phillion] In this article, it was determined that four wavefront sensors in a square geometry would be sufficient to correct the mirror aberrations in the telescope. These simulations included the expected residual noise from the atmosphere of $200 \mathrm{~nm}$ rms error for atmospheric turbulence integrated over 15 seconds. As a result of this work, the baseline configuration of the LSST focal plane array was chose to contain four wavefront sensors within the 3.5 degree field-of-view as shown in Fig. 2. 


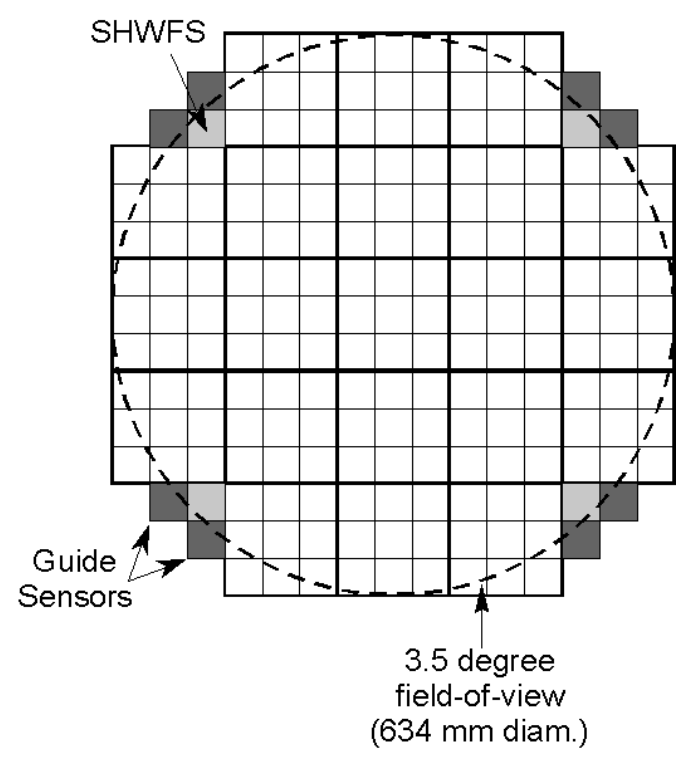

Fig. 2 Layout of the LSST focal plane array. The focal plane of the telescope is primarily populated with CCD arrays performing the telescope surveys. There are four SSD arrays, labeled Shack-Hartmann wavefront sensors(SHWFS), located in four corners of the array, primarily within the 3.5 degree field-of-view of the telescope. There are also eight guide sensors adjacent to the curvature wave-front sensors but outside of the target science field.

In this article we investigate the use of Shack-Hartmann wavefront sensors to measure the mirror deformations. Curvature sensors have also been proposed for wave-front sensing on LSST, however, the implementation of the curvature senor envisioned for the LSST requires one to deal with many issues which the Shack-Hartmann wavefront sensors, SHWS, are immune to such as overlapping pupil images, problems with spiders, registering the intra and extra focal length images, normalizing their total intensities and correcting for the vignetting differences between the two stars used for the two images. In addition the curvature sensor requires that the CCD array be manufactured with two levels representing the intra and extra-focal images. As such these CCDs would be different from the science array and would not be interchangeable.[curvature paper] The Shack-Hartmann wavefront sensor envisioned for the LSST uses a lens placed below the focal plane to reimage the pupil of the telescope onto a lenslet array and a field stop at the focal plane which would limit the field-of-view and prevent signal from adjacent lenslets contaminating one another. The advantages of this approach are the overall simplicity of the wave-front reconstruction, robustness of the wave-front sensor and it uses the same CCD arrays as the science focal plane array. There are several possible implementations of the Shack-Hartmann wave-front sensor. For each of these implementations it is envisioned that a reimaging lens and lenslet array would be placed past the focal plane with the wave-front sensor CCD array approximately $2 \mathrm{~cm}$ beyond the science focal plane array as we discuss in detail below. For each of the possible implementations, the wave-front sensor CCD array would be stationary and would be the same chip design as the science array CCDs. In one case, the moving SHWS, there would be a single Shack-Hartmann wave-front sensor in each of the four wave-front sensor locations that would move, the reimaging lens and lenslet array, in a plane parallel to the focal plane and pick suitable stars to use for the wave-front reconstruction. In another case, stationary array of SHWS, there would an array of stationary ShackHartmann wave-front sensors, $8 \times 8$, in each of the four wave-front sensor locations that would increase the effective field-of-view thereby "seeing" suitable stars to use for the wave-front reconstruction without requiring any motion of the wave-front sensor. The final case would be a hybrid of the two in which case a smaller array, 7x7, of Shack-Hartmann wave-front sensors could be implemented in each of the four wave-front sensor locations and moved around only when needed to find a suitable star. The advantages of the single SHWS would be that it would always be able to find a suitable star but the disadvantages would be that it would require moving the wave-front sensor with each repointing of the telescope. The advantages of the stationary array of SHWS would be that it would require no moving parts but the disadvantages with this approach is the need for a large array of wave-front sensors to increase the field-of-view and a decrease in performance in the most challenging case of looking at the Galactic pole in the $U$ band where stars are the scarcest. The advantages of the hybrid array are that for the vast majority of cases the SHWS could remain stationary and would not be required to move, however, in the case where the telescope is pointing at the galactic pole and looking in the $\mathrm{U}$ band for instance the wave-front sensor could be moved to find a suitable star for a wave-front reconstruction 
with a low residual variance. The motion of the hybrid array would be much simpler than the moving SHWFS and could be accomplished with only 4 discreet positions in $\mathrm{x}$ and four discreet positions in y to provide a total on sky coverage area of over 136 square arcminutes.

\section{SHACK-HARTMANN IMPLEMENTATION ON THE LSST}

A Shack-Hartmann wave-front sensor is composed of a lenslet array placed in the pupil plane of the optical system being measured. The lenslet array is composed of an array of identical lenslets, each of which measures a small portion of the optical beam. A CCD camera is placed one focal length, f.l., behind the lenslet array in the far-field. A plane wave incident on the lenslet array produces an array of focal spots located on the optical axis of the individual lenslets. An aberrated beam produces a local gradient in the phase, $\nabla \phi$, across each of the lenslets, displacing the focal spots from the optical axis as shown in Figure 3. Each individual spot is displaced from the center of the lenslet by a distance $\mathrm{s}=(\nabla \varphi)(\mathrm{f} .1.) \lambda / 2 \pi$, where $\lambda$ is the wavelength of the incident light, as illustrated in Figure 4. Therefore, the ShackHartmann wave-front sensor measures the wave-front gradient with a spatial resolution equal to the sub-aperture size. At a minimum, a quad cell is required to measure the two-dimensional wave-front tilt.

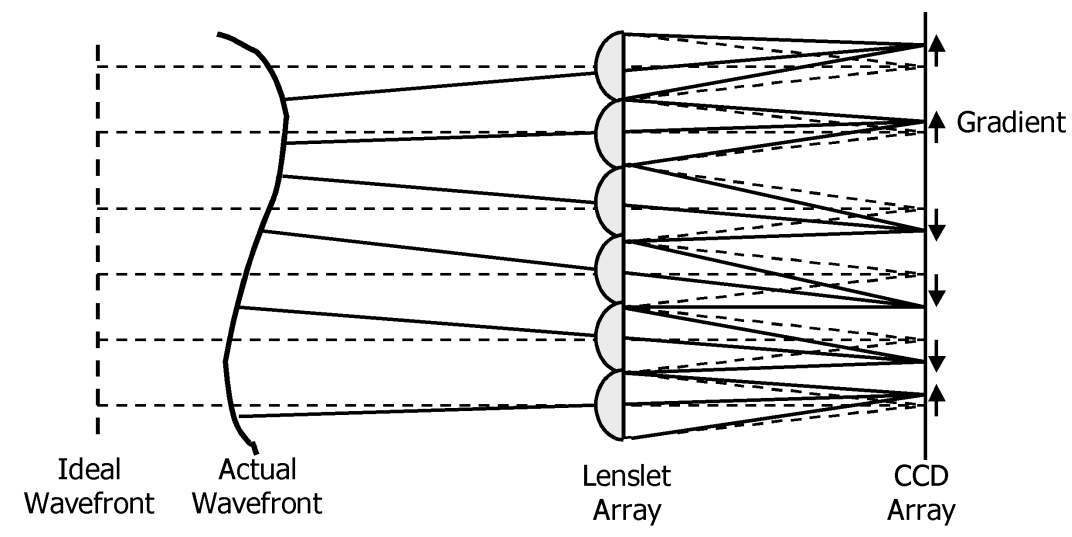

Fig. 3. Illustration denoting hypothetical Hartmann spots from a plane wave, dashed lines, and a severely aberrated beam, solid line.

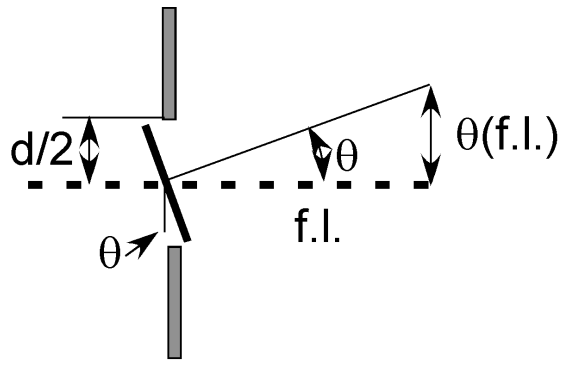

Fig. 4. Principle of operation of each lenslet. The angular displacement of the spot for each individual lenslet indicates the average slope vector of the wave-front across the given lenslet.

The schematic of the Shack-Hartmann wave-front sensor as envisioned for the LSST is shown in Figure 3. The lens in the LSST telescope section of this figure is a simplification of the LSST telescope shown in Fig. 1 and the aspheric lens in the wave-front sensor is likewise a simplification of multi-element lens shown in detail in Fig. 6. In this case a 5 $\mathrm{mm}$ effective focal length lens is used to form a $4 \mathrm{~mm}$ image of the LSST pupil onto a lenslet array. The wave-front $\mathrm{CCD}$ array is at a plane approximately $2 \mathrm{~cm}$ past the science focal plane array but uses the same CCD arrays as are used in the science focal plane array. The lenslet array is chosen to consist of $\mathrm{f} / 4$ lenslets to increase the field-of-view for each of the subapertues. A square field stop of (14.25 degrees) $(5 \mathrm{~mm})$ or $1.25 \mathrm{~mm}$ by $1.25 \mathrm{~mm}$ is placed at the focus of the 
LSST telescope such that signal from adjacent $\mathrm{f} / 4$ lenslets do not contaminate one another. This field stop represents an area on the sky of 0.174 square arcminutes which then limits the area over which each sensor will see. The moving version of the wave-front sensor would have one of these Shack-Hartmann wave-front sensors in each of the four sections reserved for wave-front sensing and the reimaging lens and lenslet array would move to find a suitable star. The stationary array version would contain an array of 64 Shack-Hartmann wave-front sensors for an overall sky coverage at each of the four sections of 11.1 square arcminutes. The hybrid array version would contain an array of 49 ShackHartmann wave-front sensors for an overall sky coverage at each of the four sections of 8.5 square arcminutes. Due to the field stop linear dimension of $1.25 \mathrm{~mm}$ and the effective linear dimension of the reimaging lens of $5 \mathrm{~mm}$ or four times the field stop size, the motion of the hybrid array would be much simpler than the moving SHWFS and could be accomplished with only 4 discreet positions in $\mathrm{x}$ and four discreet positions in y to provide a total on sky coverage area of over 136 square arcminutes in each of the four sections. Pushing the lenslets to operate at $f / 2$ would increase the sky coverage by more than a factor of four.

For the case of 8 subapertures across the LSST pupil, each lenslet would represent $1.05 \mathrm{~m}$ at the pupil plane. The turbulence would then cause the Hartmann spots to have a FWHM of $\sim \lambda \mathrm{f} / \#\left(\mathrm{~d} / \mathrm{r}_{\mathrm{o}}\right)=(0.5 \mu \mathrm{m})(4)(1 \mathrm{~m} / 0.15 \mathrm{~m})=13 \mu \mathrm{m}$ where $f / \#=4$ is the focal length of the lenslet divided by the length of one of its sides, $\lambda$ is the wavelength and $r_{o}$ is the Fried parameter at that wavelength. The Fried parameter is proportional to $\lambda^{6 / 5}$ making the Hartmann spots only weakly dependent on the wavelength. In the U band the FWHM of the Hartmann spots would be $\sim 14 \mu \mathrm{m}$ and in the Y band they would be $\sim 12 \mu \mathrm{m}$, which is slightly larger than the CCD pixel size of $10 \mu \mathrm{m}$. This represents a good compromise between the necessity of the Hartmann spots to be greater than a pixel width on the CCD but also in minimizing the number of CCD pixels that collect signal from the Hartmann spots to thereby increase the signal-to-noise ratio of the measurement. At $\mathrm{f} / 4$, the lenslets would have a pitch of 500 microns and a focal length of $2 \mathrm{~mm}$.

\section{LSST Telescope}

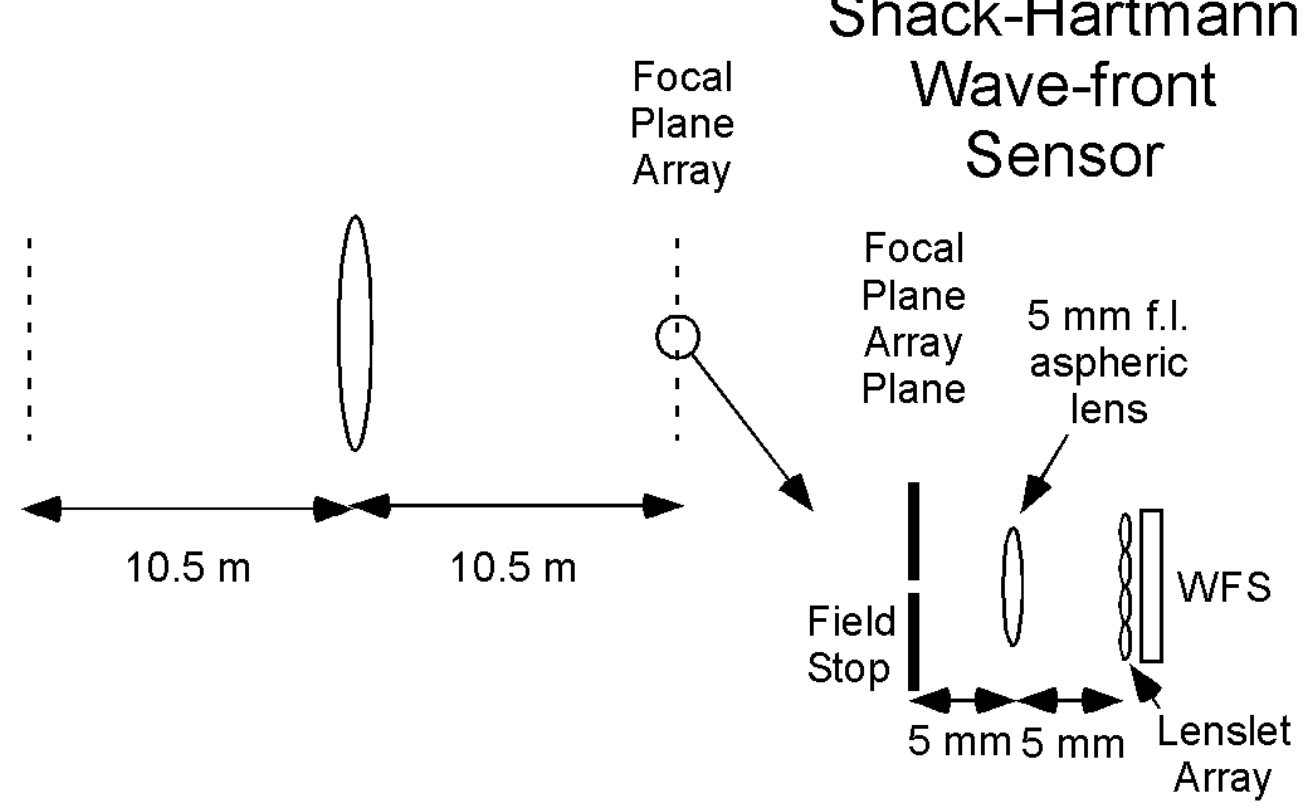

Fig. 5. Schematic of the Shack-Hartmann wavefront sensor (SHWFS) located on the focal plane array. On the left side of the figure the LSST telescope is drawn as a lens focusing the light onto the focal plane array. The Shack-Hartmann wave-front sensor on the right side of the figure has a field stop at the focal plane to prevent signal from adjacent lenslets contaminating one another. A short focal length, $5 \mathrm{~mm}$, aspheric lens is then used to form an image of the telescope pupil onto a lenslet array which then forms the Hartmann spots on the wave-front sensor CCD array.

The lens used to form an image of the LSST pupil onto the lenslet array was designed to have a short focal length to minimize the offset between science focal plane array and the Shack-Hartmann wave-front sensor and to maximize the 
number of such sensors that could be located within the active wave-front control area within the focal plane array. The lens is an $\mathrm{f} / 1.25,5.0 \mathrm{~mm}$ EFL, which has a focal plane coverage of $1.25 \mathrm{~mm}$ square focal plane coverage, 0.41 arcmin., with near diffraction limited performance over the $330-1060 \mathrm{~nm}$ spectral band. The lens is telecentric at FP and does not require any lens motion or focal plane shift for filter changes. The lens is composed of two triplet lenses in which the first surface of the first triplet and both outer surfaces of the last triplet are aspheres. The length between the lenslet array and the focal plane is approximately $19 \mathrm{~mm}$. The lens gives good performance at $200 \mathrm{lp} / \mathrm{mm}$. Possible ways to increase the sky field-of-view would be to increase the lenslet arrays to $f / 3$ or $f / 2$ such that the light would fall onto fewer pixels and so that the field-of-view of the reimaging lens would be increased. At $\mathrm{f} / 4$ with a $5 \mathrm{~mm}$ f.l.lens, the area that is seen on the sky is $5 . / 4$. or $1.25 \mathrm{~mm}$ square which is 125 by 125 pixels which is 0.4167 arcmin. by 0.4167 arcmin. or $0.17 \mathrm{sq}$. arcmin. At $\mathrm{f} / 3$ with a $5 \mathrm{~mm}$ f.l.lens, the area that is seen on the sky is $5 . / 3$. or $1.67 \mathrm{~mm}$ square which is 167 by 167 pixels which is 0.56 arcmin. by 0.56 arcmin. or $0.31 \mathrm{sq}$. arcmin. At $\mathrm{f} / 2$ with a $5 \mathrm{~mm}$ f.l.lens, the area that is seen on the sky is $5 . / 2$. or $2.5 \mathrm{~mm}$ square which is 250 by 250 pixels which is $0.83 \mathrm{arcmin}$. by $0.83 \mathrm{arcmin}$. or $0.69 \mathrm{sq}$. $\operatorname{arcmin}$. As the $\mathrm{f} / \#$ of the reimaging lens is increased it will likely take up more area in which case the gains would be diminished due to the inability to pack as many lenses in the 42 by $42 \mathrm{~mm}$ area reserved for the Shack-Hartmann wave-front sensor. Need to get the lens to function in the $U$ band as well and know what the transmission through the lens will be in each of the bands as that will effect the fluence. Currently the lens is designed for a resolution of 200 lines $/ \mathrm{mm}$ which is 5 microns. The FWHM of the atmospherically degraded spots from LSST will be 1.22 (lambda/ro)(f.l.) or 1.22lambdaf/\#(D/ro) with ro being proportional to $(\text { lambda })^{\wedge} 1.2$. At lambda $=500 \mathrm{~nm}$, ro $\sim 17.2 \mathrm{~cm}$ such that the spot size FWHM will be approximately $1.22(0.5 \mathrm{um})(1.25)(8.4 / 0.172)=37$ microns. That means that the achievable resolution of the lens can be between 10 and 20 microns, or 50 to 100 lines $/ \mathrm{mm}$, and not strongly affect the blurring.

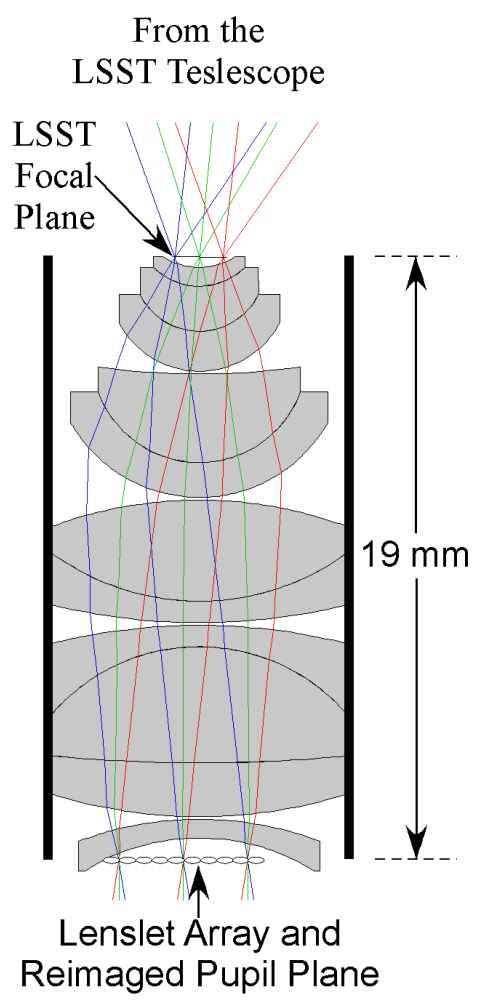

Figure 6 Raytrace of a reimaging lens used to reform the pupil of the LSST telescope onto a lenslet array. The lens design has a nominal focal length of $5 \mathrm{~mm}$ and achieves a resolution of 200 lines per $\mathrm{mm}(5$ microns $)$ over all of the LSST bands.

The Shack-Hartmann diagnostic can be calibrated in a straightforward manner using a fiber-coupled light source with a small prism or mirror affixed to the end of the fiber to direct the light from the focal plane of the telescope into the reimaging lens and through the lenslet array. These can be done for at each of the filter bands and the fixture will easily fit between the final focusing lens and the focal plane array. 


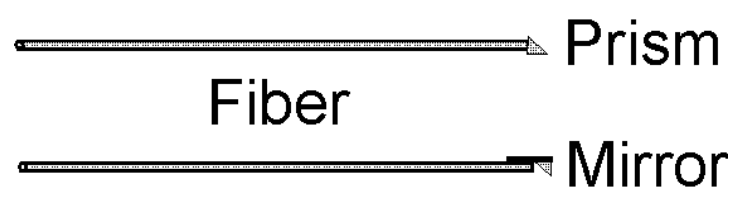

Fig. 7 Calibration of the Shack-Hartmann wave-front sensor.

\section{AVAILABILITY OF STARS AND EXPECTED FLUENCE LEVELS}

The focal plane array for the LSST will contain 3.5 gigapixels and acquire an image of the night sky every 15 seconds. Each pixel in the focal plane array will be $10 \mu \mathrm{m}$ square, representing a 0.2 arcsecond patch of sky. The pixels will have a full well capacity of 100,000 e-, a read noise of $<5$ e- rms, a dynamic range of 16 bits and a dark signal of $2 \mathrm{e}-/ \mathrm{sec}\left(95^{\text {th }}\right.$ percentile). LSST will operate with six different wavelength filter bands; U(330-400 nm), G(402-552 nm), R(552-691 $\mathrm{nm}), \mathrm{I}(691-818 \mathrm{~nm}), \mathrm{Z}(818-922 \mathrm{~nm})$ and $\mathrm{Y}(950-1060 \mathrm{~nm})$. The overall transport efficiency of photons through the camera and electrons detected in the focal plane array for each of the filter bands is expected to be $40 \%$ in the $U$ band, $68 \%$ in the $\mathrm{G}$ band, $79 \%$ in the $\mathrm{R}$ and $\mathrm{I}$ bands, $72 \%$ in the $\mathrm{Z}$ band and $25 \%$ in the $\mathrm{Y}$ band. The camera consists of the three optical lenses and coatings, the filter with coatings, the quantum efficiency of the detector and the fill factor for the detector, $91 \%$. The expected transmission through the telescope, reflection from the three mirrors, for each of the filter bands is expected to be $74 \%$ in the $\mathrm{U}$ band, $88 \%$ in the $\mathrm{G}$ band, $87 \%$ in the $\mathrm{R}$ band, $89 \%$ in the $\mathrm{I}$ band and $90 \%$ in the $\mathrm{Z}$ and $\mathrm{Y}$ bands. The overall transport efficiency of photons through the telescope and camera and electrons detected in the focal plane array is therefore expected to be 0.30 in the $\mathrm{U}$ band, 0.59 in the $\mathrm{G}$ band, 0.69 in the $\mathrm{R}$ band, 0.71 in the $\mathrm{I}$ band, 0.65 in the $\mathrm{Z}$ band and 0.23 in the $\mathrm{Y}$ band.

For the LSST, a requirement was established that each of the four wave-front sensor locations would have an rms error of less than $200 \mathrm{~nm} 95 \%$ of the time. This value of $200 \mathrm{~nm}$ was set by the measured level of residual atmospheric turbulence over the 15 second integration period of the telescope, $\sim 200 \mathrm{~nm}$. To determine how well the Shack-Hartmann wave-front sensor will function it is necessary to calculate the probability that a star greater than magnitude, m, will be observed within a given field-of-view of the sensor. The probability, $\mathrm{P}(>\mathrm{m} \mid \mathrm{A})$ that a given star of magnitude greater than $\mathrm{m}$ will be in a given area of sky, A, can be expressed by

$$
P(>m \mid A)=1-\exp (-A N\langle>m\rangle),
$$

where $\mathrm{N}(>\mathrm{m})$ is the number of stars brighter than $\mathrm{m}$ in a given area of sky. $\mathrm{N}(>\mathrm{m})$ is a strong function of magnitude, $\mathrm{m}$, and galactic coordinates and has its lowest value at the galactic poles. The most challenging case for the ShackHartmann wave-front sensor will therefore be when the telescope is pointed at the galactic pole and in particular when the telescope is operating in the $\mathrm{U}$ band where the availability of stars is the lowest. 

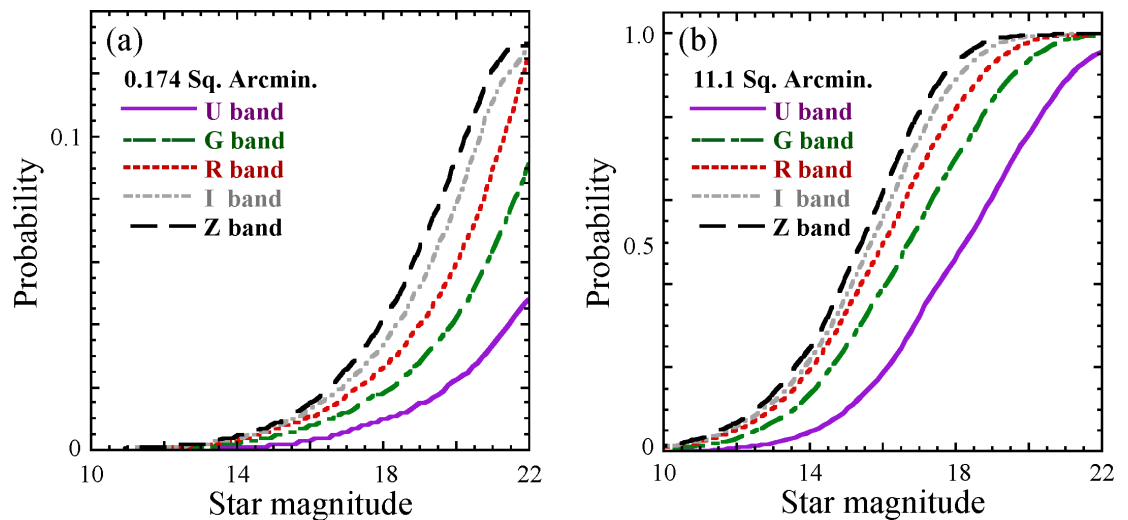

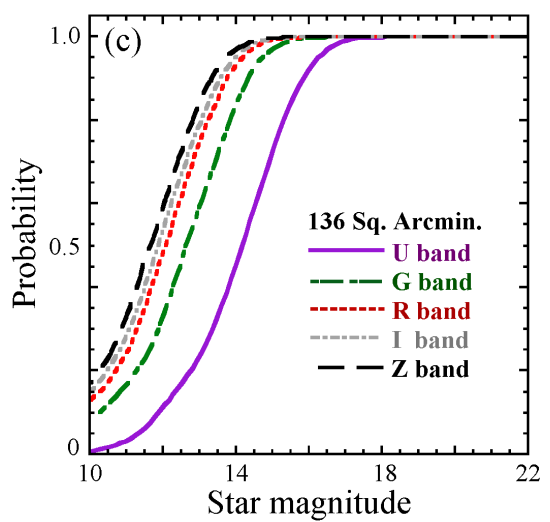

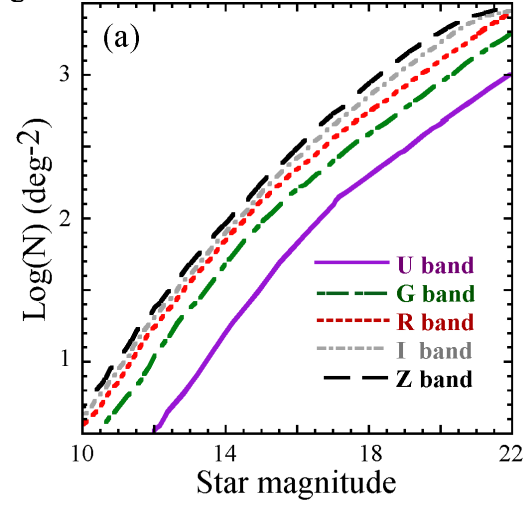
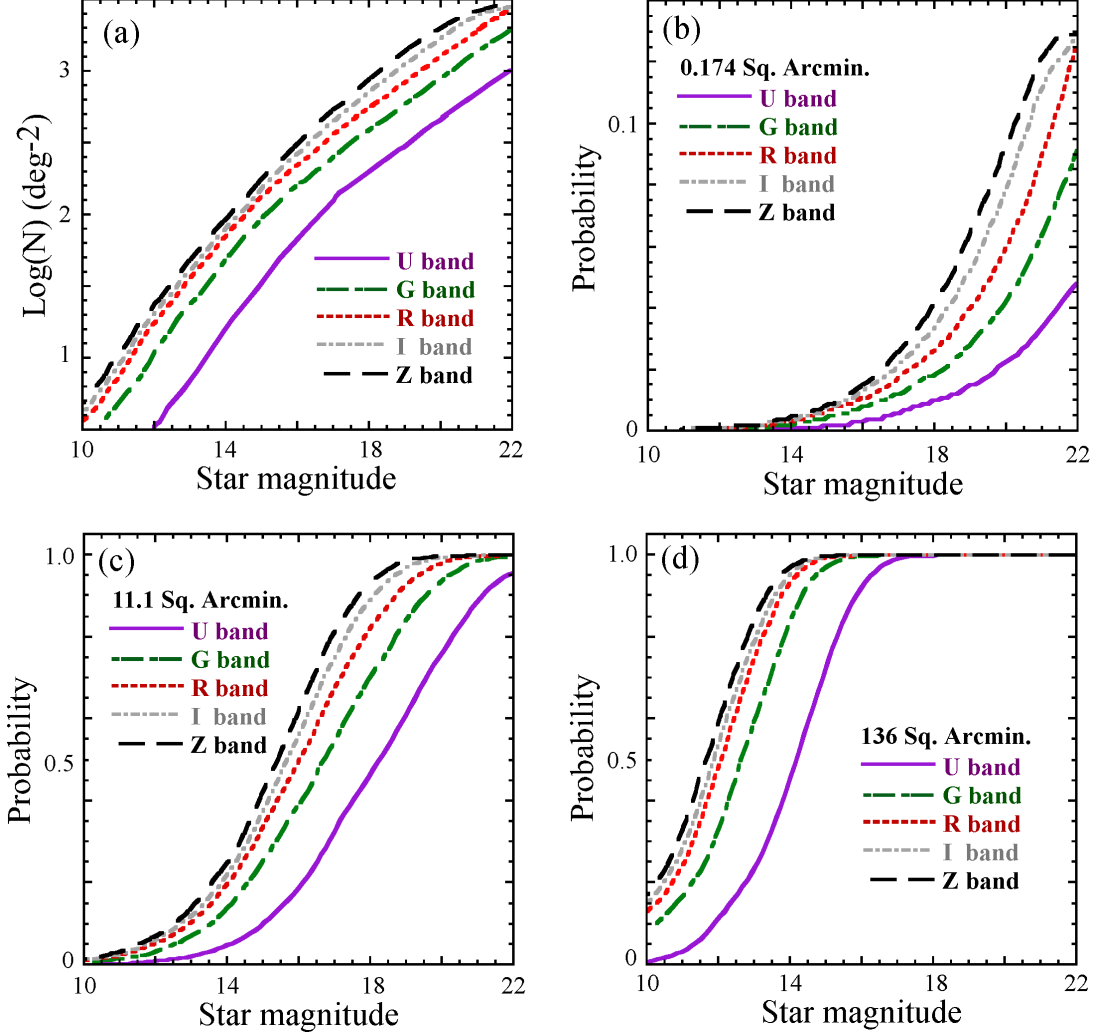

Figure 8 Probability of finding a star brighter than magnitude $m$, in five sloan digital sky survey bands, at the north galactic pole in sq. arcmin. Fig. 8a represents the probability over an area of 0.174 sq. arcmin. corresponding to a single SHWFS. Fig. $8 \mathrm{~b}$ represents the probability over an area of 11.1 sq. arcmin. corresponding to a fixed array of $8 \mathrm{x} 8$ SHWFS. Fig. 8c represents the probability over an area of $136 \mathrm{sq}$. arcmin. corresponding to the hybrid SHWFS with an array of 7x7 SHWFS and also to the moving SHWFS. For an area of 11.1 sq. arcmin., the fixed array, there is a $95 \%$ probability of detecting a star brighter than magnitude 21.8 in the $U$ band or an $80 \%$ probability of detecting a star brighter than magnitude 20.2 in the U band which would be the most difficult case for the Shack-Hartmann wave-front sensor. For an area of 136 sq. arcmin., the hybrid array, there is a $95 \%$ probability of detecting a star brighter than magnitude 16.2 in the U band (Derived from star counts, Zeljko "The photon rates, stellar density and SNR calculations.”)

For a magnitude 20 star, the expected number of electron-hole pairs created in the Shack-Hartmann wave-front sensor would be $\sim 2600 \mathrm{e}-\mathrm{h} / \mathrm{sec}$ in the R band including the telescope/CCD throughput of 0.69 and a spectral bandwidth of 0.14 microns or $\sim 40,000$ electron-hole pairs. That results in $\sim 1300 \mathrm{e}-\mathrm{h} / \mathrm{subap}$ over the $15 \mathrm{~second}$ integration time. A magnitude 21 star would produce $\sim 500 \mathrm{e}-\mathrm{h} / \mathrm{subap}$ and a magnitude $22 \mathrm{star} \sim 200 \mathrm{e}-\mathrm{h} / \mathrm{subap}$. In the U band, the ShackHartmann wave-front sensor will capture $\sim 8600$ electron-hole pairs for a magnitude 20 star with approximately $\sim 280$ e$\mathrm{h}$ /subap. . In the Y band, the Shack-Hartmann wave-front sensor will capture the least number of photons at $\sim 4300$ 
electron-hole pairs for a magnitude 20 star with approximately $\sim 140 \mathrm{e}-\mathrm{h} / \mathrm{subap}$ due in part to the lower quantum efficiency of the detector at this wavelength range. Given a full well capacity of 100,000 electrons and assuming that the individual Hartmann spots are distributed over four pixels, the CCD will saturate at the magnitudes of 13.6, 15.7, 15.2, 14.7, 14.1, 12.8 for the U, G, R, I, Z and Y bands, respectively.

The median V sky brightness levels in mag./arcsec ${ }^{2}$ for the different filter bands are 22.74 in the U band, 21.65 in the $\mathrm{G}$ band, 21.03 in the R band, 19.97 in the I band, 18.95 in the $\mathrm{Z}$ band and 18 in the $\mathrm{Y}$ band.[ Simulated Survey Technical Analysis Report for opsim1.29, pg. 46] The median airmass expected for each of the filter bands is 1.3.[ Simulated Survey Technical Analysis Report for opsim1.29, pg. 62] This field stop in front of each Hartmann sensor represents an area on the sky of 0.174 square arcminutes which then limits the area over which each sensor will see and over which sky background noise influence the measurement. The sky background from the 0.174 square arcminute patch of sky will then be spread over roughly half of the 50 x 50 CCD pixels beneath each of the Hartmann sensors.

A $10 \mu \mathrm{m}$ pixel in the focal plane array represents $0.2 \operatorname{arcsec}$ on the sky. The reimaging lens and lenslet array combination result in a $10 \mu \mathrm{m}$ pixel on the wave-front sensor array representing $0.5 \operatorname{arcsec}$ on the sky. Therefore, a $2 \times 2$ area on the wave-front sensor would see a patch of sky that was 1 arcsec by 1 arcsec and would see a sky background of magnitude are 22.74 in the $\mathrm{U}$ band, 21.65 in the G band, 21.03 in the R band, 19.97 in the I band, 18.95 in the $\mathrm{Z}$ band and 18 in the Y band. A $200 \mathrm{~nm}$ rms error requires an approximate signal-to-noise ratio of 2.25 , which represents about 2 magnitudes in star brightness over the background magnitude. As such one can easily see from Fig. 8 above, given the expected sky background noise levels, that a Shack-Hartmann sensor that travels over the entire 136 sq. arcminutes or the hybrid array that moves in four discreet locations in $\mathrm{x}$ and $\mathrm{y}$ would easily satisfy the conditions for the LSST telescope.

\section{WAVE-FRONT RECONSTRUCTION ALGORITHM}

For the Shack-Hartmann wave-front sensor implementation on LSST we chose to reconstruct the wave-front using an iterative modal wave-front reconstruction. The modal reconstruction component expands the measured wave-front into a set of basis functions, Zernike polynomials in this article, and solves for the coefficients of the set of basis functions which best fit the measured wave-front gradients. The phase, $\phi(r, \theta)$ can then be expresses as a sum of Zernike polynomials, $Z(r, \theta)$,

$$
\phi(r, \theta)=\sum_{i=1}^{k} \alpha_{i} Z_{i}(r, \theta),
$$

where $r$ and $\theta$ represent the radius and azimuthal angle in polar coordinates and $\alpha$ represents the coefficient in front of each of the Zernike modes. The lenslet array sections the LSST pupil into square subapertures denoted by the subscript $\mathrm{j}$. In each of these $\mathrm{j}$ subapertures the wavefront gradient is measured in two orthogonal directions, $\partial \phi / \partial x$ and $\partial \phi / \partial y$. These measured wave-front gradients then represent the sum of the gradients of the Zernike modes within this $\mathrm{j}$ subaperture or

$$
\begin{gathered}
\left.\frac{\partial \phi(r, \theta)}{\partial x}\right|_{j}=\left.\sum_{i=1}^{k} \alpha_{i} \frac{\partial Z_{i}(r, \theta)}{\partial x}\right|_{j} \\
\left.\frac{\partial \phi(r, \theta)}{\partial y}\right|_{j}=\left.\sum_{i=1}^{k} \alpha_{i} \frac{\partial Z_{i}(r, \theta)}{\partial y}\right|_{j}
\end{gathered}
$$

This can be expressed in matrix form as

$$
\Phi=\mathrm{ZA}
$$


where $\Phi$ represents the vector of measured wave-front gradients in each of the subapertures, $Z$ represents the matrix of calculated Zernike derivatives within the given subapertures and A represents coefficients for each of the Zernike modes. In vector form, $\Phi$ is represented as

$$
\Phi=\left[\begin{array}{c}
\frac{\partial \phi(r, \theta)_{1}}{\partial x} \\
\frac{\partial \phi(r, \theta)_{2}}{\partial x} \\
\vdots \\
\frac{\partial \phi(r, \theta)_{j}}{\partial x} \\
\frac{\partial \phi(r, \theta)_{1}}{\partial y} \\
\frac{\partial \phi(r, \theta)_{2}}{\partial y} \\
\vdots \\
\frac{\partial \phi(r, \theta)_{j}}{\partial y}
\end{array}\right]
$$

the matrix of calculated Zernike derivatives within the given subapertures, $\mathrm{Z}$, is expressed as

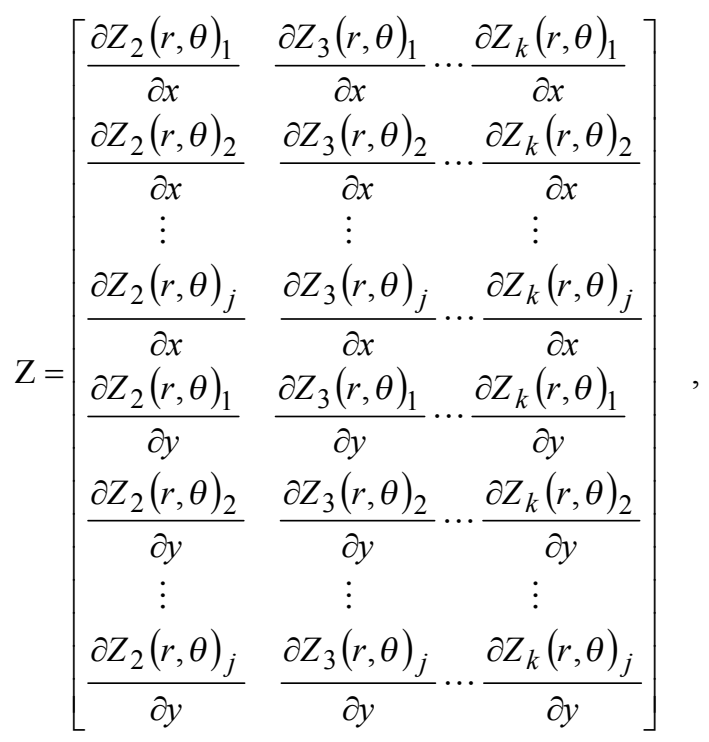

and the coefficients for each of the Zernike modes, A, can be written as

$$
\mathrm{A}=\left[\begin{array}{c}
\alpha_{1} \\
\alpha_{2} \\
\vdots \\
\alpha_{k}
\end{array}\right]
$$


The iterative component to the reconstruction is similar to a technique developed to iteratively reconstruct large phase aberrations with a Fourier or Multigrid reconstructor for metrology applications. The iterative component of the reconstruction process is very similar to a closed-loop adaptive optics system. A flow chart of the algorithm is shown in Figure 9. The primary difference between the closed-loop application and the iterative reconstruction process is that rather than driving the solution to the reference centroid locations with the use of a deformable mirror, the phase is driven to the initial measured centroid locations using a simulation loop. This is accomplished numerically by using the reconstructed phase to form simulated Shack-Hartmann spots on a simulated wave-front sensor camera and then to subtract the gradients $\mathrm{x}, \mathrm{y}_{\text {slopes }}$, derived from the numerically formed spots from the gradients derived from the initial measured spots. This can be expressed in equation form by

$$
\mathrm{x}, \mathrm{y}_{\text {slopes }, \mathrm{n}}=\left\{\begin{array}{ll}
\mathrm{x}, \mathrm{y}_{\text {slopes }, 0} & \mathrm{n}=0 \\
\mathrm{x}, \mathrm{y}_{\text {slopes }, \mathrm{n}}-\mathrm{x}, \mathrm{y}_{\text {slopes }, 0} & \mathrm{n}>0
\end{array},\right.
$$

where $\mathrm{n}$ represents the iteration number. Specifically $\mathrm{n}=0$ represents the "measured" wave-front slopes and $\mathrm{n}>0$ represents subsequent simulation steps within the iterative loop. Although the initial measured Hartmann spots contain detector noise, no detector noise is added to the simulated Hartmann spots within the iterative loop. The composite wave-front, $\phi_{\text {composite, }}$, is then equal to the initial wave-front, $\phi_{0}=\sum_{i=1}^{k} \alpha_{i, 0} Z_{i, 0}(r, \theta)$, subtracted from the sum of the wavefronts reconstructed within the iterative loop, $\sum_{n=1}^{m} \sum_{i=1}^{k} g \alpha_{i, n} Z_{i, n}(r, \theta)$, or

$$
\phi_{\text {composite }}=\phi_{0}-\sum_{n=1}^{m} \sum_{i=1}^{k} g \alpha_{i, n} Z_{i, n}(r, \theta) \text {, }
$$

where $g$ is the percentage of the Zernike polynomial coefficients added to the composite phase for each iteration. For the simulations described below the gain term, $g$, was set to unity. In this approach the simulated Hartmann spot locations are driven towards the measured spot locations and hence the reconstructed wave-front is driven to the initial wave-front being measured. 


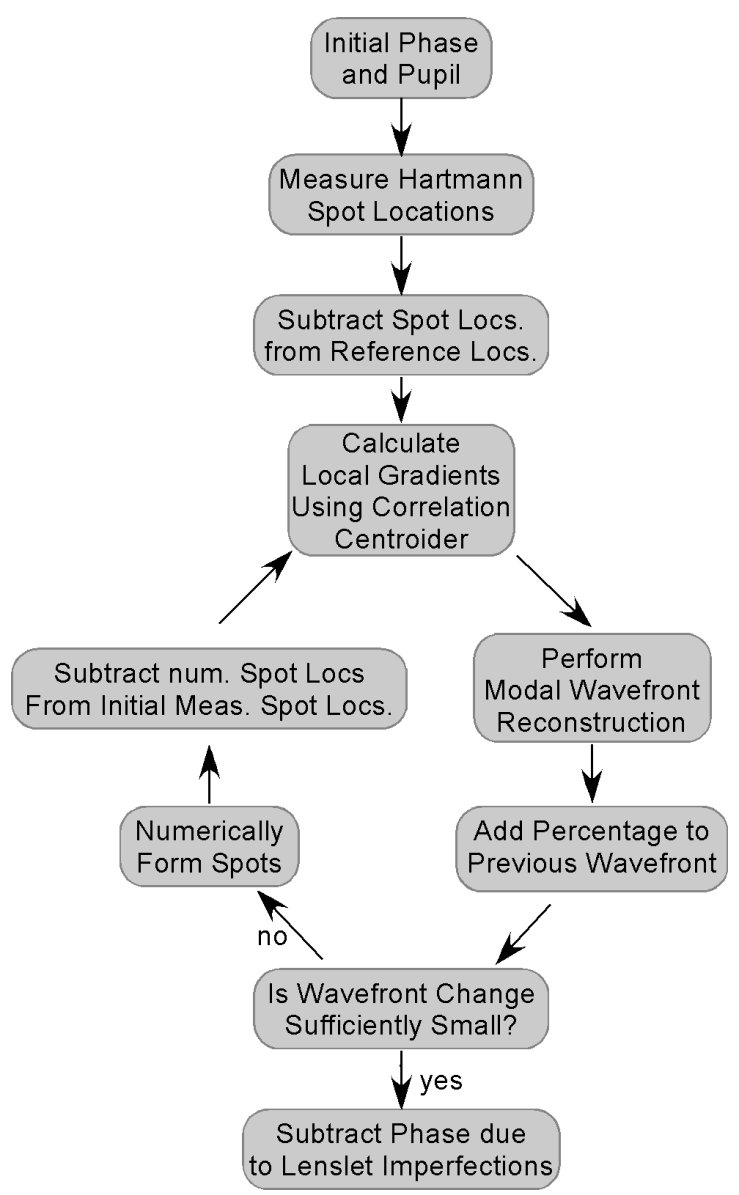

Fig. 9 Flowchart for the open-loop iterative application.

The final step in the process would then be to subtract the contribution to the phase caused by imperfections in the lenslet array. This would be accomplished by sending in a nearly flat wave-front into the wave-front sensor and calculating the apparent reconstructed phase assuming that the reference spots are located exactly on a regular array on the detector. This "reference" wave-front would then be subtracted from the wave-fronts calculated using the iterative reconstruction method.

\section{SIMULATION RESULTS AND DISCUSSION}

If the source of illumination for the Hartmann sensor is a point source, as in a traditional Hartmann sensor, then the spot displacements can be found using a number of centroiding techniques including center-of-mass, dynamic windowing, thresholding, cross-correlation, weighted center-of-mass and iterative weighted center-of-mass.[] If there is an extended scene illuminating the pupil, however, then the scene displacements at each of the sub-apertures is measured by performing cross-correlations between the scenes in the separate sub-apertures. This latter technique was pioneered by the solar adaptive optics community[] and is the technique used in this article to handle cases of both point source illumination and extended scene illumination.

Need to address expected noise levels and if you have too many stars which saturate your sensor

Show performance for minimal star magnitude expected

From curvature paper the mean sky brightness is expected to be [u_21.8,g_21.3,r_20.8,i_19.7,z_17.5,y_17.3] $\mathrm{U}(330-400 \mathrm{~nm}), \mathrm{G}(402-552 \mathrm{~nm}), \mathrm{R}(552-691 \mathrm{~nm}), \mathrm{I}(691-818 \mathrm{~nm}), \mathrm{Z}(818-922 \mathrm{~nm})$ and $\mathrm{Y}(950-1060 \mathrm{~nm})$. 
In principle I would have to run the simulations as a function of star magnitudes for each of the bands and demonstrate that everything is Kosher or at least the magnitude level at which the residual turbulence exceeds the $200 \mathrm{~nm}$ level. -

Closed loop tests of the algorithm with a single mirror(deformable) model (?)

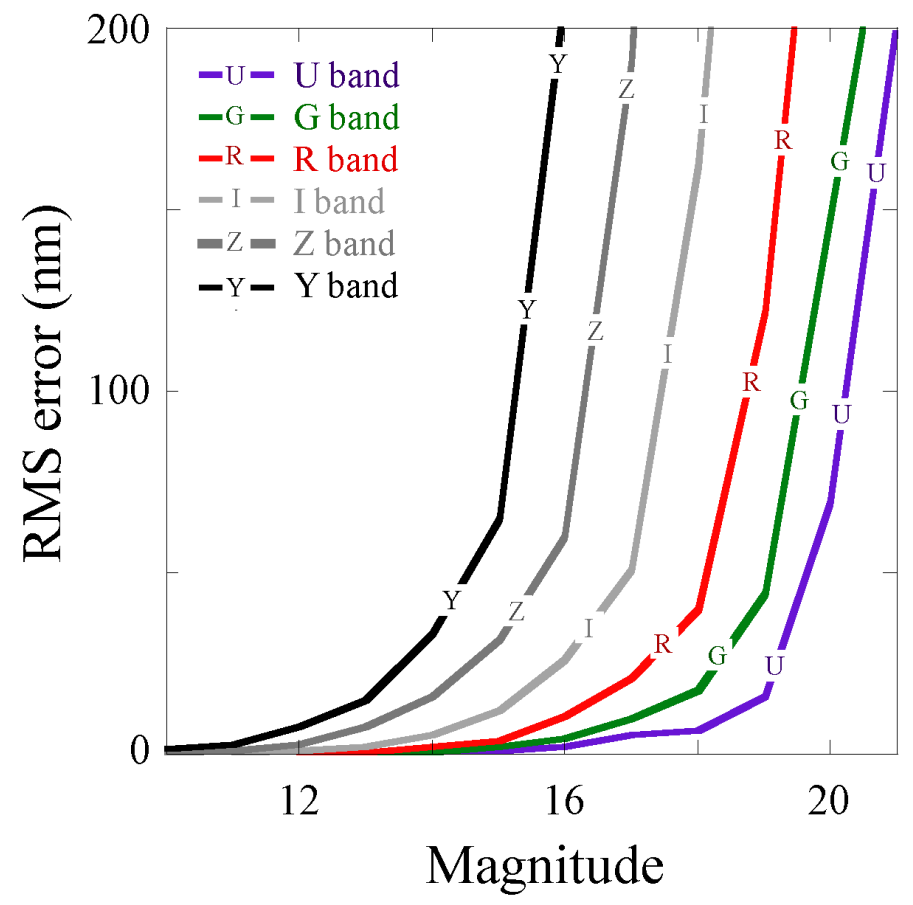

Fig. 10 Expected reconstruction RMS error as a function of observation bands and star magnitude.

These results indicate that the observational bands will reach an error equivalent to the expected residual atmospheric turbulence, $200 \mathrm{~nm}$, reconstructing from stars of magnitude 21, 20.5, 19.5, 18.2, 17 and 16 for the U, G, R, I, Z and Y bands, respectively. This represents a magnitude between 1.15 to 2 brighter than the median $\mathrm{V}$ sky brightness levels in mag./arcsec ${ }^{2}$ for the different filter bands.

This then allows one to assess the probability that each of the Hartmann wave-front sensors could meet the desired requirements of the LSST telescope that the wave-front sensors would function for greater than $95 \%$ of the measurements. As such the star probability was calculated at the equatorial poles where the fewest stars are present and hence where this requirement is the most difficult to achieve. Given the required star magnitudes in fig. 10 and the expected probabilities given in Fig. 8 one can evaluate the three Hartmann sensor configurations proposed. For the single Hartmann sensor that moves over the entire area allocated to the wave-front sensor and a hybrid array that moves in four discreet positions in $\mathrm{x}$ and $\mathrm{y}$, both can access an on-sky area of greater than 136 sq. arcmin. and hence would fall under the probabilities listed in Fig. 8c. In this case there is a greater than $95 \%$ chance of seeing a star brighter than magnitude 16.2, 14.7, 14.2, 13.9, 13.6 and 13.4 for the U, G, R, I, Z and Y bands, respectively. The probability of seeing a star greater than that required to achieve a reconstruction error of $200 \mathrm{~nm}$ would be essentially $100 \%$ of the time in all of the bands at the Galactic pole which is the most stringent case..

The fixed array of Hartmann sensors, which does not move around the wave-front sensor location, has access to an on-sky area that is sixteen times smaller or an area of 11.1 sq. arcmin. and hence the probability curves shown in Fig. 8b are applicable. In this case there is a greater than $95 \%$ chance of seeing a star brighter than magnitude 21.8, 20.2, 19.3, 18.6, 18.2 and 18 for the U, G, R, I, Z and Y bands, respectively. The probability of seeing a star greater than that required to achieve a reconstruction error of $200 \mathrm{~nm}$ would be met $88 \%, 97 \%, 96 \%, 91 \%, 81 \%$ and $64 \%$ of the time for the U, G, R, I, Z and Y bands, respectively. Considering this is at the galactic pole this would represent a small fraction of the observational space over which LSST would operate. The hybrid array which could move in four discreet locations in $\mathrm{x}$ and $\mathrm{y}$ would then represent a good compromise. For the vast majority of measurements the hybrid wavefront sensor would not require any movements. For observations near the galactic pole the wave-front sensor would 
require repositioning $\sim 40 \%$ of the time in the $\mathrm{Y}$ band to ensure a reconstruction error less than $200 \mathrm{~nm}$. In addition, this assumes a reconstruction from only one of the wave-front sensors. Reconstruction from multiple wave-front sensors which use dimmer stars could be averaged together to yield a reconstructed error that is less than the $200 \mathrm{~nm}$ requirement as well.

\section{SUMMARY}

This paper describes the methodology for measuring the wide-field-of-view LSST telescope aberrations using ShackHartmann wave-front sensors located in the four corners of the camera focal plane to enable active control of the mirrors. We presented designs for the implementation of several Shack-Hartmann wave-front sensors which could be implemented on the LSST. These designs include a SHWFS that moves within the WFS section and uses catalogued stars of appropriate brightness, an array of SHWFS within the WFS section which use the large number of sensors to increase the field-of-view and does not require motion of the SHWFS and a hybrid array that is capable of discreet motion, with four positions in $\mathrm{x}$ and four positions in $\mathrm{y}$, when looking close to the galactic pole where the density of stars is lowest. We presented a new wave-front reconstruction technique for Shack-Hartmann wave-front sensors, iterative modal reconstruction, and demonstrated that this technique was effective in reconstructing the wave-front on the LSST pupil. We simulated the expected performance of the Hartmann sensors with the reconstructor under the expected conditions in which they would operate on the LSST and demonstrated that two of the Hartmann wave-front sensors, a moving single Hartmann sensor and a hybrid array of Hartmann sensors which could move in four discreet locations in $\mathrm{x}$ and y, would meet the LSST performance goals of $200 \mathrm{~nm} \mathrm{rms}$ wave-front error over $95 \%$ of observations. The fixed array of Hartmann sensors was shown to achieve the LSST performance goals of $200 \mathrm{~nm}$ rms wave-front error over $95 \%$ of observations over the vast majority of observational locations. The most stringent observational location, the galactic pole, met the $200 \mathrm{~nm}$ residual error requirement greater than $60 \%$ of the time in each of the observational bands. Sufficient numbers of reference stars required for the wave-front sensor operation were shown to be statistically available for any LSST wavelength band and zenith pointing angle. A comprehensive analysis of phase reconstruction errors was performed and the performance goals were achieved without any averaging to decrease sources of noise. In the case of the stationary array of Hartmann sensors or for the hybrid array the reconstructed phase from multiple SHWFS could be averaged together to greatly reduce the residual atmospheric aberrations and further improve the estimation of the telescope aberrations. In addition, implementation of the hybrid array would require motion of the wave-front sensor for only a small fraction of observations.

\section{ACKNOWLEDGEMENTS}

The authors acknowledge useful discussions with W. De Vries, B. Bauman, S. Olivier and C. Carrano. LSST is a public-private partnership. Funding for design and development activity comes from the National Science Foundation, private donations, grants to universities, and in-kind support at Department of Energy laboratories and other LSSTC Institutional Members. This work is supported by in part the National Science Foundation under Scientific Program Order No. 9 (AST-0551161) and Scientific Program Order No. 1 (AST-0244680) through Cooperative Agreement AST-0132798. Portions of this work are supported by the Department of Energy under contract DE-AC02-76SF00515 with the Stanford Linear Accelerator Center, contract DE-AC02-98CH10886 with Brookhaven National Laboratory, and with the Lawrence Livermore National Laboratory under the auspices of the U.S. Department of Energy under Contract DE-AC52-07NA27344.

Additional funding comes from private donations, grants to universities, and in-kind support at Department of Energy laboratories and other LSSTC Institutional Members.

\section{REFERENCES}

